\title{
Medical Image of the Week: Sleep Bruxism
}



Figure 1. Hypnogram and polysomnographic tracing showing an episode of rhythmic masticatory muscle activity (RMMA) during sleep. RMMA is defined when at least 3 consecutive EMG bursts (frequency $1 \mathrm{~Hz}$ ) lasting greater than or equal to 0.25 seconds are scored on the masseter and temporalis channels.

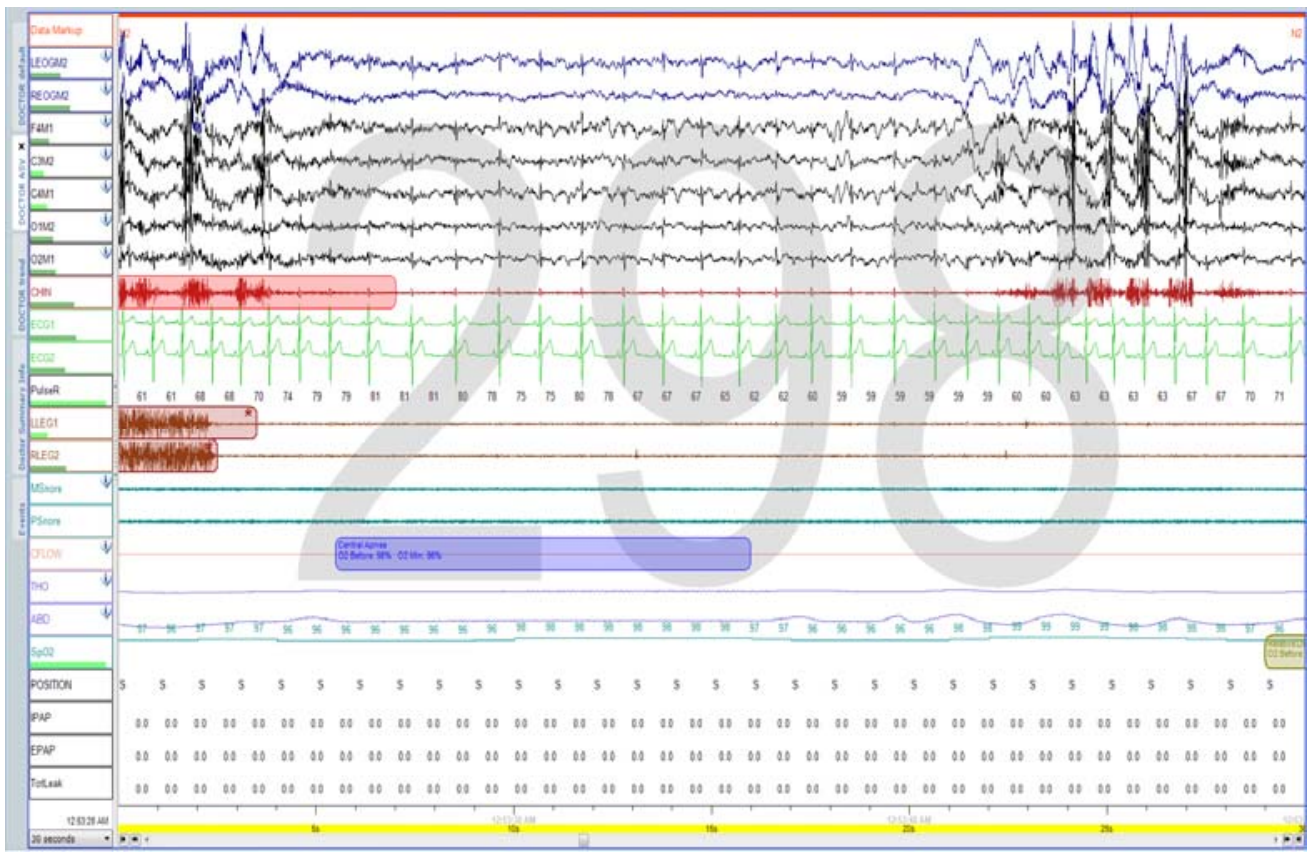

Figure 2. Thirty second epoch of polysomnogram showing phasic sleep-bruxism during stage N2 sleep. 
A 42 year-old man with a past medical history of insomnia, post-traumatic stress disorder, depression and both migraine and tension headaches was referred for an overnight sleep study. He had presented to the sleep clinic with symptoms of obstructive sleep apnea. Medications included sumatriptan, amitryptiline, sertraline, and trazodone. His sleep study showed: sleep efficiency of $58.2 \%$, apnea-hypopnea index of 33 events per hour, and arousal index of 14.5/hr. Periodic limb movement index was 29.2/hr. The time spent in the sleep stages included N1 (3.6\%), N2 (72.5\%), N3 (12.9\%), and REM (10.9\%). Figure 1 is representative of the several brief waveforms seen on his EEG and chin EMG. Sleep bruxism (SB) is a type of sleep-related movement disorder that is characterized by involuntary masticatory muscle contraction resulting in grinding and clenching of the teeth and typically associated with arousals from sleep $(1,2)$.

The American academy of sleep medicine (AASM) criteria for sleep related bruxism diagnosis:

1. The patient reports or is aware of tooth-grinding sounds or tooth clenching during sleep.

2. One or more of the following is present: A. Abnormal wear of the teeth; B. Jaw muscle discomfort, fatigue, or pain and jaw lock upon awakening; and C. Masseter muscle hypertrophy upon voluntary forceful clenching.

3. The jaw muscle activity is not better explained by another current sleep disorder, medical or neurological disorder, medication use, or substance use disorder.

The exact etiology of SB is unknown. It is associated with sleep arousals, genetic factors, stress, anxiety and behavioral factors and medications like selective serotonin receptor inhibitors, tobacco, alcohol and recreational drug use and sleep disordered breathing (2).

The electromyogram (EMG) activity pattern in patients with SB is known as rhythmic masticatory muscle activity (RMMA) and involves the masseter and temporalis muscles in patterns of phasic and/or tonic contractions, most typically during stages N1 and N2 of sleep $(2,3)$. Clinically, bruxism can result in abnormal tooth wear, masseter muscle hypertrophy, reduced salivation, and morning headaches $(1,2)$.

Sleep bruxism has been shown to be strongly associated with tension and migraine headaches (4). Treatment of the underlying sleep disordered breathing with positive airway pressure may eliminate bruxism during sleep (5). Treatment involves oral appliances such as occlusal splints or mandibular advancement devices (2). There is insufficient evidence to support pharmacotherapy in the treatment of sleep bruxism (1).

Jared Bartell ${ }^{1}$, Safal Shetty, $\mathrm{MD}^{1,2}$, and John Roehrs, $\mathrm{MD}^{1,2}$

${ }^{1}$ University of Arizona Medical Center, Tucson, AZ

${ }^{2}$ Southern Arizona VA Health Care System, Tucson, AZ 


\section{References}

1. Macedo CR, Macedo EC, Torloni MR, Silva AB, Prado GF. Pharmacotherapy for sleep bruxism. Cochrane Database Syst Rev. 2014;10:CD005578. [CrossRef] [PubMed]

2. Carra MC, Huynh N, Lavigne G. Sleep bruxism: a comprehensive overview for the dental clinician interested in sleep medicine. Dent Clin North Am. 2012;56(2):387413. [CrossRef] [PubMed]

3. Valiente López M, van Selms MK, van der Zaag J, Hamburger HL, Lobbezoo F. Do sleep hygiene measures and progressive muscle relaxation influence sleep bruxism? Report of a randomised controlled trial. J Oral Rehabil. 2014 Nov 21. [CrossRef] [PubMed]

4. De Luca Canto G, Singh V, Bigal ME, Major PW, Flores-Mir C. Association between tension-type headache and migraine with sleep bruxism: a systematic review. Headache. 2014;54(9):1460-9. [CrossRef] [PubMed]

5. Oksenberg A, Arons E. Sleep bruxism related to obstructive sleep apnea: the effect of continuous positive airway pressure. Sleep Med. 2002;3(6):513-5. [CrossRef] [PubMed] 